\title{
Ab Initio MR-CISD Study of Gas-phase Basicity of Formamide in the First Excited Singlet State
}

Ivana Antol, Mirjana Eckert-Maksic, and Hans Lischka

Cartesian coordinates [a.u] and total energies [a.u] for optimized ground state and first excited state structures of formamide, $\mathrm{N}$-protonated and $\mathrm{O}$-protonated formamide.

$\begin{array}{lrrr}\begin{array}{l}\text { MP2/cc-pVTZ } \\ \text { ground state }\end{array} & & & \\ \text { Formamide 1 } & & & \\ \text { C1 } & 0.00000000 & 0.79208816 & 0.00000000 \\ \text { O1 } & 2.26766569 & 0.43485433 & 0.00000000 \\ \text { N1 } & -1.77792805 & -1.05817672 & 0.00000000 \\ \text { H1 } & -0.84749682 & 2.69008373 & 0.00000000 \\ \text { H2 } & -1.22470694 & -2.87294875 & 0.00000000 \\ \text { H3 } & -3.62363679 & -0.64126156 & 0.00000000\end{array}$

$E_{\text {tot }}=-169.60545$ a.u.

$\mathrm{N}$-protonated formamide 2

$\begin{array}{lrrr}\mathrm{C} 1 & 0.00000000 & 1.05280989 & 0.00000000 \\ \mathrm{O} 1 & -2.21852903 & 0.91705386 & 0.00000000 \\ \mathrm{~N} 1 & 1.54882710 & -1.46097184 & 0.00000000 \\ \mathrm{H} 1 & 1.24822080 & 2.69459829 & 0.00000000 \\ \mathrm{H} 2 & 0.29655850 & -2.93726180 & 0.00000000 \\ \mathrm{H} 3 & 2.68083163 & -1.59191096 & 1.56454206 \\ \mathrm{H} 4 & 2.68083163 & -1.59191096 & -1.56454206\end{array}$

$E_{\mathrm{tot}}=-169.90910$ a.u.

O-protonated formamide 3

$\begin{array}{lrrr}\mathrm{C} 1 & 0.00000000 & 0.80332825 & 0.00000000 \\ \mathrm{~N} 1 & 2.08666961 & -0.46155427 & 0.00000000 \\ \mathrm{O} 1 & -2.11722270 & -0.40365117 & 0.00000000 \\ \mathrm{H} 1 & 0.06361196 & 2.85012275 & 0.00000000 \\ \mathrm{H} 2 & 2.07003813 & -2.37756649 & 0.00000000 \\ \mathrm{H} 3 & 3.76481634 & 0.44809942 & 0.00000000 \\ \mathrm{H} 4 & -3.56736641 & 0.71946598 & 0.00000000\end{array}$

$E_{\text {tot }}=-169.93563$ a.u. 
CASSCF/aug-cc-pVDZ

ground state

Formamide 1

$\begin{array}{lrrr}\text { C1 } & -0.00180199 & 0.80296490 & 0.00000006 \\ \mathrm{~N} 1 & -1.78214283 & -1.06885967 & 0.00000000 \\ \mathrm{O} 1 & 2.28578615 & 0.45569611 & -0.00000009 \\ \mathrm{H} 1 & -0.81613777 & 2.69819395 & -0.00000017 \\ \mathrm{H} 2 & -1.24777943 & -2.87201897 & -0.00000005 \\ \mathrm{H} 3 & -3.61303155 & -0.65699737 & 0.00000001\end{array}$

$E_{\text {tot }}=-169.05088$ a.u.

$\mathrm{N}$-protonated formamide 2

$\begin{array}{lrrr}\text { C1 } & -0.00610286 & 1.06858412 & -0.00000008 \\ \text { N1 } & 1.55333376 & -1.47206141 & 0.00000019 \\ \text { O1 } & -2.24296705 & 0.94477406 & 0.00000082 \\ \text { H1 } & 1.24276590 & 2.69224753 & -0.00000094 \\ \text { H2 } & 0.33727903 & -2.95150425 & -0.00000051 \\ \text { H3 } & 2.67416692 & -1.59646289 & 1.54766999 \\ \text { H4 } & 2.67416707 & -1.59646175 & -1.54766953 \\ E_{\text {tot }}=-169.36580 \text { a.u. } & & & \end{array}$

O-protonated formamide 3

$\begin{array}{lrrr}\mathrm{C} 1 & -0.01065265 & 0.81025098 & 0.00000002 \\ \mathrm{~N} 1 & 2.10739600 & -0.47272195 & 0.00000000 \\ \mathrm{O} 1 & -2.16008767 & -0.39356693 & 0.00000005 \\ \mathrm{H} 1 & 0.05396141 & 2.85196313 & -0.00000011 \\ \mathrm{H} 2 & 2.08936056 & -2.37027827 & -0.00000012 \\ \mathrm{H} 3 & 3.76329043 & 0.44184398 & 0.00000009 \\ \mathrm{H} 4 & -3.57573990 & 0.71786034 & -0.00000006\end{array}$

$E_{\mathrm{tot}}=-169.37321$ a.u. 
CASSCF/aug-cc-pVDZ

First excited state

Formamide 4

$\begin{array}{lrrr}\text { C1 } & -0.16953397 & 1.06946185 & -0.19496965 \\ \text { N1 } & -1.98015753 & -0.94661027 & 0.14549574 \\ \text { O1 } & 1.96846214 & 0.34622142 & -1.55269238 \\ \text { H1 } & 0.26712886 & 2.22355067 & 1.43346321 \\ \text { H2 } & -1.21212598 & -2.42598712 & 1.05818999 \\ \text { H3 } & -2.62649146 & -1.54706045 & -1.53221105\end{array}$

$E_{\mathrm{tot}}=-168.88763$ a.u.

$\mathrm{N}$-protonated formamide 5

$\begin{array}{lrrr}\text { C1 } & 0.05978776 & 1.01262185 & -0.17941591 \\ \text { N1 } & 1.51122112 & -1.43623058 & 0.02493213 \\ \text { O1 } & -1.97545365 & 0.89642485 & 1.42800749 \\ \text { H1 } & 1.19951501 & 2.70579800 & -0.10285131 \\ \text { H2 } & 0.35603393 & -2.91747239 & -0.35794808 \\ \text { H3 } & 2.25793259 & -1.67836389 & 1.77591150 \\ \text { H4 } & 2.92843649 & -1.43727796 & -1.26178084\end{array}$

$E_{\text {tot }}=-169.21678$ a.u.

O-protonated formamide 6

$\begin{array}{lrrr}\text { C1 } & -0.07062924 & 1.00805968 & -0.33205998 \\ \text { N1 } & -1.89844828 & -1.10579998 & 0.10894704 \\ \text { O1 } & 2.18432671 & 0.17516284 & -1.22269347 \\ \text { H1 } & 0.02221566 & 2.30497921 & 1.23710055 \\ \text { H2 } & -1.67607624 & -2.29304003 & 1.59496317 \\ \text { H3 } & -3.31013273 & -1.47470387 & -1.12609485 \\ \text { H4 } & 2.08359567 & -0.44330100 & -2.90720468\end{array}$

$E_{\text {tot }}=-169.20121$ a.u.

O-protonated formamide 7

$\begin{array}{lrrr}\text { C1 } & -0.13766969 & 1.13312314 & -0.25783881 \\ \text { N1 } & -1.78441691 & -1.10306652 & 0.02407547 \\ \text { O1 } & 1.91898938 & 0.43044640 & -1.62888871 \\ \text { H1 } & 0.00700612 & 2.29197875 & 1.41464134 \\ \text { H2 } & -1.37706870 & -2.49152597 & 1.27783488 \\ \text { H3 } & -3.29946524 & -1.33513028 & -1.11841745 \\ \text { H4 } & 3.22371932 & 1.65370518 & -1.46979392\end{array}$

$E_{\text {tot }}=-169.20503$ a.u. 


\section{MR-CISD/aug-cc-pVDZ}

ground state

Formamide 1

$\begin{array}{lrrr}\text { C1 } & 0.00143961 & 0.80055836 & 0.00000004 \\ \mathrm{~N} 1 & -1.78195910 & -1.06637163 & -0.00000001 \\ \mathrm{O} 1 & 2.26942651 & 0.44223076 & -0.00000013 \\ \mathrm{H} 1 & -0.82441482 & 2.71414056 & 0.00000002 \\ \mathrm{H} 2 & -1.23281194 & -2.88359726 & 0.00000010 \\ \mathrm{H} 3 & -3.62957567 & -0.65185853 & -0.00000006\end{array}$

$E_{\text {tot }}=-169.43836$ a.u.

$\mathrm{N}$-protonated formamide $\mathbf{2}$

$\begin{array}{lrrr}\mathrm{C} 1 & 0.02408098 & 1.03263470 & 0.00000002 \\ \mathrm{~N} 1 & 1.53676475 & -1.44649565 & -0.00000027 \\ \mathrm{O} 1 & -2.20749906 & 0.89355857 & -0.00000136 \\ \mathrm{H} 1 & 1.24497065 & 2.70615847 & 0.00000213 \\ \mathrm{H} 2 & 0.28660042 & -2.92477923 & 0.00000104 \\ \mathrm{H} 3 & 2.67080553 & -1.58446591 & 1.56269090 \\ \mathrm{H} 4 & 2.67080493 & -1.58446783 & -1.56269172\end{array}$

$E_{\mathrm{tot}}=-169.74652$ a.u.

O-protonated formamide 3

$\begin{array}{lrrr}\mathrm{C} 1 & -0.00360782 & 0.80853375 & -0.00000003 \\ \mathrm{~N} 1 & 2.09330235 & -0.46565702 & 0.00000000 \\ \mathrm{O} 1 & -2.12635956 & -0.39857233 & 0.00000002 \\ \mathrm{H} 1 & 0.05863278 & 2.86734344 & -0.00000001 \\ \mathrm{H} 2 & 2.07445089 & -2.38243299 & -0.00000001 \\ \mathrm{H} 3 & 3.77463956 & 0.44062667 & 0.00000014 \\ \mathrm{H} 4 & -3.57854409 & 0.70353601 & 0.00000006\end{array}$

$E_{\text {tot }}=-169.77223$ a.u. 
MR-CISD/aug-cc-pVDZ

First excited state

Formamide 4

$\begin{array}{lrrr}\mathrm{C} 1 & -0.18483602 & 1.06054250 & -0.18267271 \\ \mathrm{~N} 1 & -1.96132914 & -0.92152354 & 0.12325772 \\ \mathrm{O} 1 & 1.84767468 & 0.35966199 & -1.62846587 \\ \mathrm{H} 1 & 0.30968827 & 2.17352857 & 1.48160111 \\ \mathrm{H} 2 & -1.26043088 & -2.38383193 & 1.15376795 \\ \mathrm{H} 3 & -2.53247409 & -1.59881553 & -1.57305857 \\ E_{\text {tot }}=-169.27879 \text { a.u. } & & \end{array}$

$\mathrm{N}$-protonated formamide $\mathbf{5}$

$\begin{array}{lrrr}\mathrm{C} 1 & 0.06953703 & 0.97775896 & -0.16763361 \\ \mathrm{~N} 1 & 1.49821084 & -1.41467720 & 0.02494964 \\ \mathrm{O} 1 & -1.92741669 & 0.88398544 & 1.42333941 \\ \mathrm{H} 1 & 1.20514290 & 2.70091861 & -0.14128132 \\ \mathrm{H} 2 & 0.34759052 & -2.92172893 & -0.37503942 \\ \mathrm{H} 3 & 2.24713223 & -1.68938907 & 1.79570974 \\ \mathrm{H} 4 & 2.94370764 & -1.42284129 & -1.26015393\end{array}$

$E_{\mathrm{tot}}=-169.60119$ a.u.

O-protonated formamide $\mathbf{6}$

$\begin{array}{lrrr}\mathrm{C} 1 & -0.08364795 & 0.97377229 & -0.31224923 \\ \mathrm{~N} 1 & -1.86048887 & -1.07942418 & 0.09884449 \\ \mathrm{O} 1 & 2.13486507 & 0.13612935 & -1.20874994 \\ \mathrm{H} 1 & -0.00554672 & 2.33753986 & 1.22518059 \\ \mathrm{H} 2 & -1.67055719 & -2.29043536 & 1.60306638 \\ \mathrm{H} 3 & -3.27646275 & -1.47088992 & -1.15855819 \\ \mathrm{H} 4 & 2.05096314 & -0.43953254 & -2.93570521\end{array}$

$E_{\text {tot }}=-169.59819$ a.u.

O-protonated formamide 7

$\begin{array}{lrrr}\text { C1 } & -0.13439251 & 1.08760591 & -0.24428580 \\ \mathrm{~N} 1 & -1.75603672 & -1.07493405 & 0.00849968 \\ \mathrm{O} 1 & 1.92522889 & 0.39195833 & -1.55058702 \\ \mathrm{H} 1 & -0.03485154 & 2.28516991 & 1.43086987 \\ \mathrm{H} 2 & -1.39573268 & -2.49628488 & 1.27893869 \\ \mathrm{H} 3 & -3.30495002 & -1.29382714 & -1.12938164 \\ \mathrm{H} 4 & 3.13150578 & 1.75289932 & -1.63095609\end{array}$

$E_{\mathrm{tot}}=-169.60284$ a.u. 